\title{
Effect of sodium orthovanadate on ovulation isolated ovarian follicles in Siberian Sturgeon (Acipenser baerii) In Vitro
}

\author{
Dmitry Balashov ${ }^{1, *}$ and Konstantin Kovalev ${ }^{1}$ \\ ${ }^{1}$ Branch for the freshwater fisheries of "VNIRO" ("VNIIPRKH"), Rybnoe, 40A, Dmitrov district, \\ Moscow oblast, 141821, Russia
}

\begin{abstract}
Effects of sodium orthovanadate on oocyte ovulation were examined during in vitro culture of Siberian sturgeon ovarian follicles from hibernating fish. It was shown that sodium orthovanadate stimulates ovulation of Siberian sturgeon oocytes in a dose-dependent manner. The stimulating or inhibitory effect of vanadate depends on the time of addition to the incubation medium. It was also shown that the stimulating effects of orthovanadate depend on the physiological status of hibernating females whose oocytes were isolated
\end{abstract}

\section{Introduction}

In recent decades anthropogenic activities have led to the sturgeon population's decline [1]. Sturgeons are valuable fish species due to their high meat and caviar quality, and hence physiological status and nutrition management of sturgeon broodstock is particularly important in aquaculture as it affects the eggs and larval quality [2].

Using a hormonal stimulation model in vitro of maturation and ovulation of sturgeon oocytes allows solving various fundamental and applied problems [3]. While the molecular mechanisms of maturation of fish oocytes were well understood [4,5], the ovulation process is still poorly understood. Most of the early studies on the mechanisms of ovulation in fish were mainly done on teleost fish. [6-11]. Maturation activity has been related to the action of steroid hormones on the oocytes, such as progesterone. It starts with a biochemistry cascade associated with signaling molecules such as cAMP, protein kinases, and phosphorylation subunits of G-proteins that leads to germinal vesicle breakdown (GVBD). Ovulation is a process following after GVBD.

The main trigger of ovulation is a biochemical cascade associated with cyclooxygenases (COX-1 and COX-2) and the prostaglandins synthesis from arachidonic acid. The same mechanism underlies inflammatory processes in representatives of the animal world.

Vanadium compounds are used to link cancer mechanisms. Furthermore, the role of inflammatory reactions associated with the activation of the cyclooxygenase pathway and

\footnotetext{
* Corresponding author: balashofff@gmail.com
} 
the synthesis of PGE2 from arachidonic acid helps in this cascade [12-15]. As stated above, it has a presumably important role in the ovulation process.

In early studies in fish, sodium orthovanadate induced oocyte ovulation and stimulated prostaglandin synthesis in vitro, without concomitant ovulation transduction. [16]. The experiments with oocytes of amphibians showed that orthovanadate could also induce oocyte ovulation without maturation and progesterone production [17]. The role of cyclooxygenase cascade metabolites, prostaglandins (PGs), in oocyte ovulation is not understood in sturgeons, and only limited studies have been conducted.

This study was aimed to demonstrate the effect of sodium orthovanadate on the ovulation of sturgeon oocytes in vitro depending on concentrations and time of addition.

\section{Materials and methods}

\subsection{Animal and experimental design}

The work was carried out in the period from 2015 to 2017 during winter and early spring. The farmed Siberian sturgeons used in this work were collected from the stock Konakovo Sturgeon Breeding Farm (Tver region). We used 60 females 8-10 years old who belonging to the 2005-2008 cohort. All females in the experiments have already been selected for reproduction by diagnostic ultrasound. The wintering hibernating females were kept in the concrete pools with stream water. The temperature of the water was in the range of $4-8^{\circ} \mathrm{C}$. In the experimental period, fish were caught and exposed by biopsy of ovarian follicles and marked of microchips. For isolation of ovarian follicles was used a metallic probe.

\subsection{Incubation of oocytes in vitro}

Isolated follicles were washed off the Ringer solution modified for sturgeon fish (RMS) [18] and then placed in plastic boxes with L-15 medium (Sigma, U.S.A.) diluted to $70 \%$ and contained $0,75 \mathrm{~g} / \mathrm{L}$ of sodium bicarbonate and $50 \mu \mathrm{g} / \mathrm{mL}$ of gentamicin. In this solution, follicles were delivered to the laboratory (VNIIPRKh, Moscow oblast) that took about 3.5-4 h.

In the laboratory, follicles were washed off from L-15 by RMS and placed in the ventilated Petri dishes for incubations. Petri dishes of the diameter $60 \mathrm{~mm}$ contained $10 \mathrm{ml}$ RMS (it was included $0,75 \mathrm{~g} / \mathrm{L}$ sodium bicarbonate and gentamicin $50 \mu \mathrm{g} / \mathrm{mL}$ ). In every dish, we put $33 \pm 3$ ovarian follicles and introduced $10 \mu \mathrm{l}$ alcohol solution of the progesterone (Sigma Chemicals, U.S.A.) that the progesterone's final concentration in the culture medium was $333 \mathrm{ng} / \mathrm{ml}$. The follicles of each female were incubated in four-ten replications. It took stock the quantity of ovulated oocytes into each dish. Ovulation was estimated by counting the follicle envelopes separated from oocytes. The ovulation time was determined in the $\tau_{0}\left(\tau_{0}\right.$ is a dimensionless developmental rate characteristic; for detail, see [19]). Ovulation oocytes were counted through 38-40 $\tau_{0}$ after adding progesterone. $\tau_{0}$ at $16{ }^{\circ} \mathrm{C}$ for Siberian sturgeon was taken $59 \min [20]$.

Sodium orthovanadate for studying effects of concentration was added at $22 \tau_{0}$ after adding progesterone at final concentrations $100,20,10$, and $5 \mu \mathrm{M}$.

The sodium orthovanadate for studying the time effects was added in $0,17,20,22,23$, $24,26 \tau_{0}$ at a final concentration of $20 \mu \mathrm{M}$. 


\section{Results}

As shown in Fig. $1, \mathrm{Na}_{3} \mathrm{VO}_{4}$ treatment of follicles in concentration $100 \mu \mathrm{M}$ significantly $(\mathrm{P}<0,05)$ decreased ovulation percentage than control. The percentage of ovulation significantly higher than control was obtained at a final concentration of $10 \mu \mathrm{M}$. We did not observe a significant difference in the ovulation percentage at other concentrations.

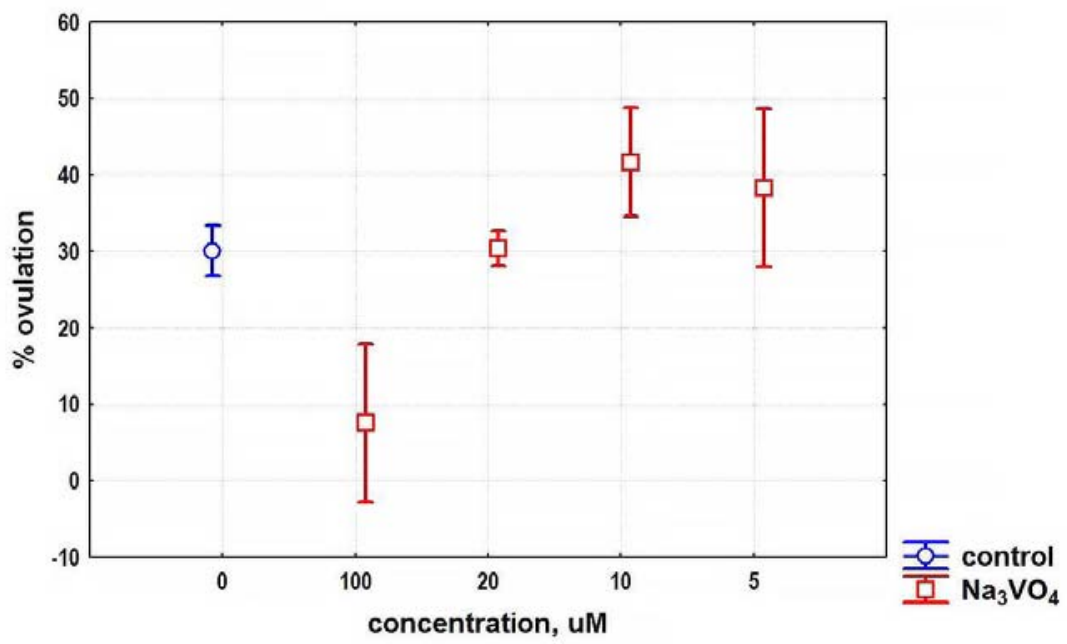

Fig. 1. Dependence of percentage of ovulation oocytes from concentrations of $\mathrm{Na}_{3} \mathrm{VO}_{4}$ (ANOVA).

The experiment findings on the effect of orthovanadate adding time on oocytes ovulation are shown in Figure 2. It was shown that adding orthovanadate simultaneously with progesterone at the start of the experiment blocks ovulation. Highly significant increase of percent oocyte ovulation relative to the control $(\mathrm{P}<0.05)$ was found after adding orthovanadate in $24 \tau_{0}$ after adding progesterone. The percent of oocyte ovulation after adding orthovanadate in 17, 20, 22, and $26 \tau_{0}$ did not significantly differ from the control.



Fig. 2. Dependence of percentage of ovulation oocytes from time of addition $\left(\tau_{0}\right) \mathrm{Na}_{3} \mathrm{VO}_{4}$ (final concentration $0,2 \mu \mathrm{M}$ ) and control (ANOVA). 
Moreover, when we analyzed the experimental data, it was found that the follicles of different females did not respond the same way to the addition of sodium orthovanadate. To demonstrate this, we introduced an additional criterion. We divided females into two groups and assigned them ranks: females with percent ovulation in control above $30 \%$ received rank 1, and females whose percent ovulation in control was below $30 \%$ received rank 2.

We corrected the experimental data regarding the assigned ranks. We found that sodium orthovanadate has a different influence on ovulation of oocytes the females with high percent ovulation and low.

As shown in figure $3 \mathrm{a}$, oocytes of females with high percent ovulation in control (rank 1) have a weak, insignificant response on low vanadate concentrations, and concentrations 20 and $100 \mu \mathrm{M}$ inhibits ovulation. However, for females whose follicles showed a low percentage of ovulation in control (rank 2), orthovanadate at a concentration of $10 \mu \mathrm{M}$ almost doubled ovulation (fig. 3b).
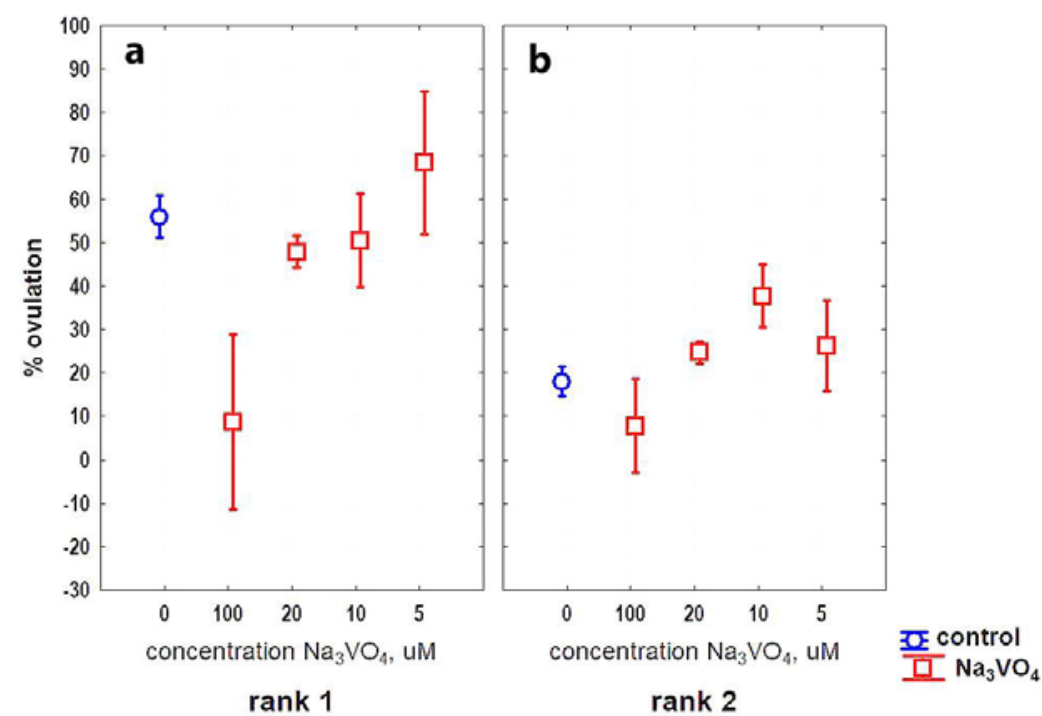

Fig. 3. Dependence of percentage ovulation oocytes from concentrations of $\mathrm{Na}_{3} \mathrm{VO}_{4}$ and rank of females (ANOVA).

We found similar results in experiments with the time of sodium orthovanadate addition. The difference that in oocytes of the first rank it significantly reduced the ovulation percentage after addition at 17 and 20 after adding progesterone. Adding orthovanadate at 22-23 $\tau_{0}$ after adding progesterone did not influence the percent of ovulation of females of rank 1 (fig. $4 \mathrm{a}$ ). Fig. $4 \mathrm{~b}$ shows the effect of orthovanadate on the percent of ovulation oocytes of the rank 2 was expressed in a small insignificant increase of percent of ovulation after adding it at 17-23 $\tau_{0}$ after addition progesterone. A significant double increase of ovulation percent was observed after adding at 24 and $26 \tau_{0}$. The addition of orthovanadate simultaneously with progesterone $\left(\begin{array}{ll}0 & \tau_{0}\end{array}\right)$ blocked ovulation regardless of the female's rank (fig.4). 

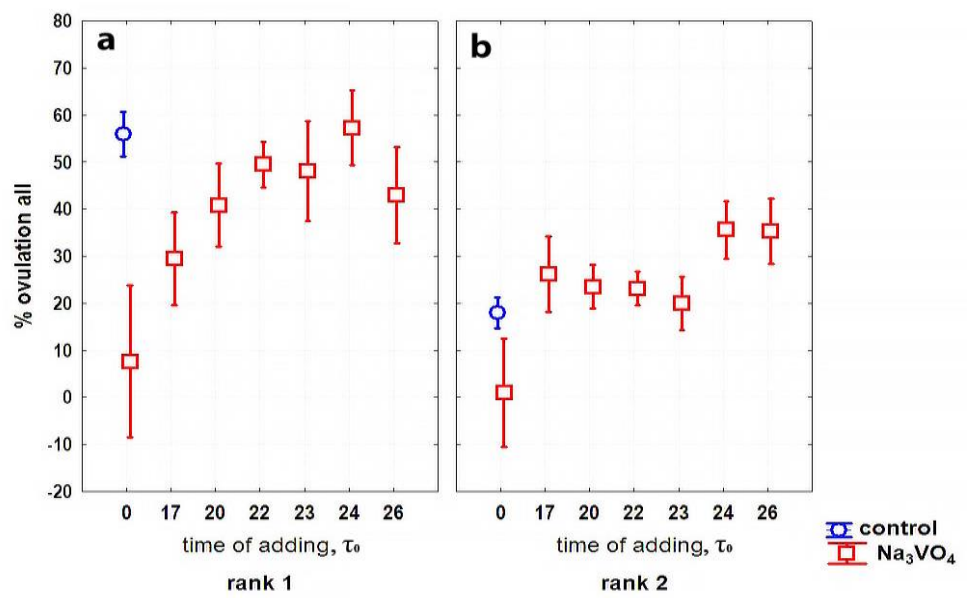

Fig. 4. Dependence of percentage ovulation oocytes from time of addition $\left(\tau_{0}\right) \mathrm{Na}_{3} \mathrm{VO}_{4}$ (final concentration $0,2 \mu \mathrm{M}$ ) and rank of females (ANOVA).

\section{Discussion}

In early studies on oocytes teleost fish, it was shown that vanadate stimulates the synthesis of prostaglandins without the release of progesterone, which is necessary for the competence of oocyte maturation. The present work demonstrated that high concentrations of sodium orthovanadate on sturgeon oocytes inhibit ovulation. In contrast, low concentrations stimulate oocyte ovulation in vitro, depending on the time of vanadate addition to the medium.

Oocyte maturation is triggered by progesterone and terminated by the GVBD. Oocyte maturation depends on the physiological state of the females and is completed at 14-18 $\tau_{0}$. Then ovulation occurs. According to our data, ovulation begins 6-8 $\tau_{0}$ after the GVBD. Also, we observed that females whose oocytes matured in vitro later than $18 \tau_{0}$, to ovulate began later, and the proportion of oocytes ovulated in vitro was much lower. It has also been shown that these traits correlate with the quality [21].

This work showed that sodium orthovanadate had little effect on the oocyte ovulation from females with a high proportion of ovulation in vitro in control. In contrast to oocytes with a low percent of ovulation in control, were significantly stimulates ovulation. Likely, the cyclooxygenase cascade processes and, as a result, the level of accumulation of prostaglandins in female oocytes may be lower, or the time points of a sufficient concentration of prostaglandins to initiate ovulation are shifted towards an increase in time. Therefore they ovulate poorly without additional stimulation with orthovanadate. This may indicate the heterogeneity of oocyte physiological status from different pre-spawning females, which influences the time and proportion of oocyte ovulation in vitro.

\section{Conclusion}

Thereby, in the present work, we tried to provide insights on the relationship between the accumulation time of prostaglandins level to initiate ovulation and the physiologic status of oocytes from females using the cyclooxygenase cycle stimulator orthovanadate.

Moreover, using a model system of hormonal stimulation in vitro of maturation and ovulation of sturgeon oocytes, it was shown that sodium orthovanadate affected sturgeons oocytes in a dose-dependent manner and only at the appointed time points. 


\section{Reference}

1. M.A. Webb and S.I. Doroshov, General and Comparative Endocrinology 170(2), 313321 (2011) doi: 10.1016/j.ygcen.2010.11.024

2. S. Ghiasi, B. Falahatkar, M. Arslan, K. Dabrowski, Animal Reproduction Science 178, 23-30 (2017) doi: 10.1016/j.anireprosci.2017.01.005

3. M.N. Skoblina and B.F. Goncharov, Russian Journal of Developmental Biology 43(3), 157-163 (2012) doi:10.1134/S1062360412030083

4. M. Conti, M. Hsieh, A.M. Zamah, J.S. Oh, Mol. Cell. Endocrinol. 356, 65-73 (2012) doi: 10.1016/j.mce.2011.11.002

5. D.L. Berlinsky, L.W. Kenter, B.J. Reading, F.W. Goetz, Fish Physiology. 38, 1-52 (2020) doi: 10.1016/bs.fp.2020.09.001

6. C. Fujimori, K. Ogiwara, A. Hagiwara, T. Takahashi, Mol. Cell. Endocrinol. 362, 7684 (2012) doi: 10.1016/j.mce.2012.05.013

7. A. Hagiwara, K. Ogiwara, Y. Katsu, T. Takahashi, Biol. Reprod. 90(6), 1-14 (2014) doi: 10.1095/biolreprod.113.115485

8. T. Takahashi, C. Fujimori, A. Hagiwara, and K. Ogiwara, Zoological science 30(4), 239-247 (2013) doi: 10.2108/zsj.30.239

9. O.M. Knight, G. Van Der Kraak, Gen. Comp. Endocrinol. 213, 50-58 (2015) doi: 10.1016/j.ygcen.2014.12.014

10. A.L. Lister, and G. Van Der Kraak, General and comparative endocrinology 159(1), 46-57 (2008) doi: 10.1016/j.ygcen.2008.07.017

11. F.W. Goetz, P. Garczynski, Fish Physiol. Biochem. 17, 33-38 (1997) doi: 10.1023/A:1007765902327

12. E. Eilati, C.C. Small, S.R. McGee, et al. Lipids Health Dis/ 12, 152 (2013) doi: $10.1186 / 1476-511 \mathrm{X}-12-152$

13. D.M. Duffy, Che Myong Ko, Misung Jo, Mats Brannstrom, Thomas E. Curry. Endocrine Reviews 40(2), 369-416 (2019) doi:10.1210/er.2018-00075

14. E. Ricciotti, and G.A. FitzGerald, Arteriosclerosis, thrombosis, and vascular biology 31(5), 986-1000 (2011) doi: 10.1161/ATVBAHA.110.207449

15. J. Korbecki, I. Baranowska-Bosiacka, I. Gutowska, R. Piotrowska, and D. Chlubek, 163(1), 103-111 (2015) doi: 10.1007/s12011-014-0176-4

16. T. Hsu and F.W. Goetz, Biol. Reprod 48, 1202-1209 (1993) doi: 10.1095/biolreprod48.6.1202

17. K.J. Chang, J.W. Kim, W.B. Im, H.M. Kang, and H.B Kwon, Journal of Experimental Zoology 277(2), 155-165 (1997) doi: 10.1002/(SICI)1097010X(19970201)277:2<155::AID-JEZ7>3.0.CO;2-O

18. B.F. Goncharov, Journal of Applied Ichthyology 18, 368-374 (2002) doi: 0175-8659/2002/1804-06-0368\$15.00/0

19. T.A. Dettlaff, A.S. Ginsburg, and O.I. Schmalhausen, Sturgeon Fishes: Developmental Biology and Aquaculture (Springer Science \& Business Media, 2012)

20. I. Lebeda, and M. Flajshans, Journal of animal science 93(8), 3759-3764 (2015) doi: 10.2527/jas.2015-9094

21. D.A. Balashov, K.V. Kovalev, N.A. Kozovkova, and A.V. Recoubratsky, Fisheries journal 5, 85-88 (2019) 